\title{
Epigenetics- The Science beyond Genetics
}

\author{
Atmakuri Shanmukha Ramya*, Sumit Majumdar, Mahesh Babu T, Baratam Srinivas, Kameswara Rao A and \\ Naga Himabindu V \\ Accredited Parliamentary Assistant, Belgium
}

Submission: January 24, 2018; Published: April 20, 2018

*Corresponding author: Atmakuri Shanmukha Ramya, Department Of Oral Pathology and Microbiology, GITAM Dental College, India, Tel: 9948581250; Email: ramya.oralpathology58@gmail.com

\begin{abstract}
Epigenetics code the information determining gene expression that is often not directly encoded in the DNA. The molecular basis of epigenetic memory remains a subject of intense debate, which is likely to arise from the collaboration of several mechanisms, including histone modifications, transcription factors and DNA methylation. Amongst all, DNA methylation is one of the key epigenetic factors involved in regulation of gene expression and genomic stability, and is biologically necessary for the maintenance of many cellular functions. This review presents the basic principles behind these epigenetic pathways and highlights the evidence suggesting that their mis-regulation which can culminate in cancer.
\end{abstract}

Keywords: Epigenetics; DNA; Genes

\section{Introduction}

The cells in a multi cellular organism have nominally identical DNA sequences, yet maintain different terminal phenotypes. This non genetic cellular memory, which records developmental and environmental cues, is the basis of epi-(above)-genetics [1,2]. The current definition of epigenetics is the study of mitotically and/or meiotically heritable changes in gene function that cannot be explained by changes in DNA sequence [3]. The term has evolved to include any process that alters gene activity without changing the DNA sequence, and leads to modifications that can be transmitted to daughter cells. Epigenetic processes are natural and essential for normal biological functions, but turbulence in their nature can ultimately lead to major adverse health and behavioral effects.

Many types of epigenetic processes have been identifiedwhich include methylation, acetylation, phosphorylation, ubiquitylation, and sumolyation [3]. Perhaps the best known epigenetic process which has been the easiest to study with existing technology, is DNA methylation.

\section{DNA Methylation}

DNA methylation was first confirmed to occur in human cancer in 1983. It involves reversible addition of a methyl group to the carbon-5 position of cytosine in cytosine-phosphateguanine (CpG) dinucleotides [4]. DNA is made up of four bases, thus there are 16 possible dinucleotide combinations that can occur. Therefore the $\mathrm{CpG}$ dinucleotide should occur with a frequency of approximately $6 \%$. However, the actual presence is only 5 to $10 \%$ of its predicted frequency [5]. The most striking feature of vertebrate DNA methylation patterns is the presence of CpG islands, that are, unmethylated GC (Guanine Cytosine) rich regions that possess high relative densities of $\mathrm{CpG}$, positioned at the promoter ends of many human genes [2,6].

In contrast to the rest of the genome, smaller regions of DNA, called CpG islands, have distinctive properties [7]. Approximately half of all the genes in humans have $\mathrm{CpG}$ islands [5]. CpG island is a region with at least 200-bp stretch of DNA which are usually located near the promoter of widely expressed genes and may often extend into the first exon [8]. The methylation and demethylation taking place in the $\mathrm{CpG}$ islands act as molecular switches, regulating gene expression [9]. A small but significant proportion of all $\mathrm{CpG}$ islands become methylated during development, and when this happens the associated gene becomes stably silent $[4,6]$. This CpG suppression may be related to the increased susceptibility of methylated cytosine to mutations [10]. Surprisingly, the human genome is not methylated uniformly but contains regions of unmethylated segments interspersed by methylated regions [5]. Developmentally programmed CpG island methylation of this kind is involved in genomic imprinting and $\mathrm{X}$ chromosome inactivation [3]. 


\section{Epigenetic Machinery}

Methylation is carried out by methylating enzymes DNMT1, DNMT3A and DNMT3B. DNMT1 involved in Maintenance methylation called Maintenance methyl transferases De novo methyltransferases DNMT3A and DNMT3B. Hypermethylation are of 2 types Maintenance and De- novo methylation based on their methylation patterns.

\section{Maintenance Methylation}

Maintenance methylation describes the processes that reproduce DNA methylation patterns between cell generations [10]. The simplest conceivable mechanism for maintenance methylation depends on semi-conservative (two copies of DNA, each containing one original strand and a new strand) copying of the parental- strand methylation pattern onto the progeny DNA strand. The methylating enzyme DNMT1 prefers to methylate those new $\mathrm{Cp}$ Gs whose partners on the parental strand already carry a methyl group [6]. Thus a pattern of methylated and nonmethylated $\mathrm{Cp}$ Gs along a DNA strand tends to be copied, and this provides a way of passing epigenetic information between cell generations. The idea that mammalian DNA methylation patterns are established in early development by De- novo methylation and then copied to somatic cells by the maintenance DNA methyltransferase. Cp G islands keep their overall unmethylated state (or methylated state) extremely stably through multiple cell generations [3]. Dramatic evidence for this maintenance mechanism comes from the finding that $\mathrm{Cp}$ G-island methylation is stably maintained even in the apparent absence of the only known maintenance DNA methyltransferase, DNMT1 [6].

\section{Denovo Methylation}

The de novo methyl transferses DNMT3A and DNMT3B are highly expressed in early embryonic cells, and it is at this stage that most programmed de novo methylation events occur. This determines the regions of the genome to be methylated and it methylates newer $\mathrm{CpG}$ regions [11]. An extreme possibility is that de novo DNA methylation in early mammalian development is an indiscriminate process potentially affecting all $\mathrm{Cp}$ Gs. Even $\mathrm{CpG}$ islands, most of which are un methylated at all times in normal cells, can acquire methylation under special developmental circumstances or in abnormal cells [6].

\section{Determination of Methylation of DNA}

Methylation of DNA can be determined by several approaches. Restriction with a methylation-sensitive enzyme generates a pattern of fragments that depends on the methylation status of the DNA [12]. The use of restriction enzymes to determine methylation status is limited to sequences that are recognized by available restriction enzymes [13]. An alternative approach to determining methylation status that is faster, and requires much less starting material, is PCR following bisulfite treatment of DNA. This technique is known as methylation- specific PCR [14]. Treatment with bisulfite converts cytosine residues to uracil residues; during $\mathrm{PCR}$, thymidine is incorporated where uracil was present in the original template DNA [12]. However, 5-methylcytosine is not altered by bisulfite treatment. Comparison of the sequence of PCR products amplified from genomic DNA and a parallel sample amplified after treatment with bisulfite demonstrates the location of 5-methylcytosine residues in the template DNA [14].

\section{DNA Methylation and Gene Regulation}

The regulation of eukaryotic gene expression is a complex process [5]. Transcription initiation is a highly controlled and integrated event that involves cis-acting and trans-acting factors. The cis-acting elements are DNA sequences that act as the substrate for the trans-acting factors, and the DNA in the vicinity is prepared for transcription [10]. Increased methylation in the promoter region of a gene leads to reduced expression, whereas methylation in the transcribed region has a variable effect on gene expression [10]. Hypomethylation and hypermethylation of DNA are relative terms and denote less or more methylation than in some standard DNA [8]. Hypomethylation usually involves repeated DNA sequences, such as long interspersed nuclear elements, whereas hypermethylation involves $\mathrm{CpG}$ islands [9]. Hypermethylation of promoter $\mathrm{CpG}$ dinucleotides results in a closed chromatin configuration (or heterochromatin), which blocks access for transcription factors to bind to the promoter region of tumor suppressor genes, resulting in their transcriptional silencing [7].

\section{DNA Hypermethylation and Cancer}

Methylation can occur physiologically and pathologically. Methylation of $\mathrm{CpG}$ islands can efficiently repress transcription of the associated gene in a manner akin to mutations and deletions and act as one of the 'hits' in the Knudsen two-hit hypothesis for tumor generation [11]. Tumor cells exhibit global hypomethylation of the genome accompanied by region-specific hyper-methylation events [15]. Through the effects of both hypo- and hyper-methylation, DNA methylation significantly affects the genomic landscape of cancer cells [15]. Reports of hyperrmethylation in cancer far outnumber the reports of hypomethylation. Many hypotheses have been proposed to explain why certain genes are methylated in cancer. One Darwinian theory suggests that particular genes become methylated in tumors because inactivation of these genes provides a selective growth advantage for the cells.

Another hypothesis suggests that genes which are under the control of PcG are more vulnerable to DNA methylation. Recently it has been shown that PcG marked genes are 20 times more likely to be methylated in cancer [11]. Hypermethylated promoters are almost always transcriptionally silent, packaged into a chromatin structure resistant to nucleases. Local cytosine methylation of a particular sequence can directly interfere with the binding of certain transcription factors [16]. Both hypermethylation (by suppressor gene inactivation) and hypomethylation (by inappropriate oncogene activation) can lead to carcinogenesis 
[12] The genes that are susceptible are those involved in cell cycle regulation (p16INK4a, p15INK4a, Rb, p14ARF) genes associated with DNA repair (BRCA1, MGMT), apoptosis (DAPK, TMS1), drug resistance, detoxification, differentiation, angiogenesis, and Metastasis. It is well established that promoter methylation can result in decreased expression of tumor suppressor genes such as p16, p15, RB, VHL, hMLH-1, APC and BRCA genes, thus contributing to increased tumorigenicity in a variety of human cancers [17].

\section{Hot Spots in P16 Gene}

Also called Cyclin-dependent kinase inhibitor 2A (CDKN2A; p16; INK4A). CpG sites act as mutational hotspots in p16 gene. Tumour suppressor gene, p16 (chromosome 9p21.3) is a cell cycle regulator involved in the inhibition of G1 phase progression in normal cells [18] p16 methylation was correlated with malignant transformation of oral epithelial dysplasia and is a potential biomarker for prediction of prognosis of mild or moderate oral epithelial dysplasia, with the overall sensitivity and specificity of $>60 \%$ [19] An alternate spliced product of the same INK4a locus is another tumor suppressor gene p14ARF (Alternative open reading frame). Interestingly, p16INK4A and p14ARF genes, transcribed from the same locus INK4A by alternate splicing, could have a diametrically opposite clinical effect in oral cancer patients when methylated [15] Promoter methylation of p16 was associated with increased disease recurrences and worse prognosis, whereas p14 methylation was strongly associated with lower disease recurrence and was found to be a good prognostic predictor for oral carcinoma [19].

\section{Cadherins}

Also called CDH1 cadherin 1, type 1 and its locus is $16 \mathrm{q} 22.1$. $\mathrm{CDH} 1$ behaves as a classical tumor suppressor gene. They help in homotypic cell adhesions and prevents invasion and metastasis of tumor cells.[4] Hypermethylation of the CDH1 promoter will lead to transcriptional silencing, which has been observed in human breast, prostate, and hepatocellular tumors. In some cases, that carry germline mutations in one allele of the CDH1 gene, the remaining allele is inactivated by DNA methylation [5] Finally, inhibition of DNA methylation can suppress the initiation of tumor development. Transcriptional silencing of CDH1 may also result from aberrant expression of transcription factors that repress its promoter [20].

\section{Connexon Gene}

Intercellular communication also occur through gap junction (GJIC) plays a significant role in maintaining tissue homeostasis by exchanging small molecules, which has long been proposed as a mechanism to regulate growth control, development and differentiation [15] Dysfunctional GJ has been recognized as being involved in carcinogenesis. Methylation has been reported to occur in the promoter region of connexon gene in various tumor cells, which is responsible for suppression of its expression.
Down regulation of expression and dysfunctional gap junctions has been shown to occur in nasopharyngeal carcinoma tissues and cells. Tan et al have reported that methylation of the promoter region of the connexon gene is likely to be an important mechanism in modulating its expression in breast cancer [17].

\section{hMLH1 Methylation}

The hMLH1 gene belongs to the human DNA mismatch repair system, and is essential in reducing the accumulation of mutations and maintaining genomic stability by repairing DNA defects [19]. Promoter methylation is one of the mechanisms responsible for the inactivation of hMLH1 protein expression and it is a potential molecular marker for OSCC. Methylation of hMLH1 is an early event, probably maintained during tumor progression. A strong association between hMLH1 methylation and OSCC was observed [16].

\section{APC Gene}

An example of a tumor suppressorgene that can be inactivated by aberrant methylation is APC (Adenomatosis Polyposis Coli). It is a tumor suppressor gene and helps in controlling the beta catenin gene and further transcription of genes. APC is mutated in $>90 \%$ of patients with familial adenomatous polyphonies coli. APC-inactivating mutations can also be demonstrated in $80 \%$ of sporadic colorectal cancers, and mutation of this gene is believed to be an early event in the natural history of colorectal cancers [3] this hypermethylation is always found on the genes where one allele is inactivated by DNA mutation, and the other is silenced through hypermethylation. These abnormalities prevent the expression of functional APC protein [14].

\section{Hypermethylation in Resected Margins of the Tumour}

Local recurrences and/or multiple primary tumors develop despite an apparently complete excision and histopathologically tumor - free surgical margins. This raised the question of molecular alterations existence in negative surgical margins, which may not be detectable by conventional microscopic histopathologic analysis, but could be detected with molecular analyses for genetic alterations associated with carcinogenesis [13] Aberrant DNA methylation was detected in normalappearing surgical margins in OSCC and HNSCC, indicating the early tumor development in surgical margins with normal histology. DAPK, E-cadherin, MGMT, p14, p16, RARbeta2 and RASSF1A genes were hypermethylated in histologically normal mucosa of head and neck tumors [11].

The age-related methylation changes observed may be associated with the long-term exposure to alcohol or tobacco and could be responsible for the epigenetic changes in a large field of oral mucosa. Hypermethylation of MGMT, DAPK1 and p16 genes promoters in cancer-free margins of HNSCC patients is frequent event that reflects the methylation status of primary tumor [13]. 


\section{Conclusion}

The area of epigenetic silencing in cancer is indeed in a state of great enthusiasm now a days. The accumulated interest for DNA methylation-based biomarkers for cancer diagnosis in the last two decades has been highly impressive. Many biomarkers are involved in cancer which can be altered due to DNA methylation. Thus, study of these interactions between genes and cancer will help to shed light on nature of the methylation defect in tumor cells and may even lead to novel therapies to reverse aberrant methylation patterns and restore growth control.

\section{References}

1. Andersen B (2010) What Is Epigenetics? Sci Translation Medicine 2: 77.

2. Weinhold B (2006) Epigenetics: The Science of Change. Environ Health Perspect 114(3): A160-A167.

3. Raghu Radhakrishnan (2011) DNA Hypermethylation as an epigenetic mark for oral cancer diagnosis. J Oral Pathol Med 40: 665-676.

4. Michael T, McCabe (2009) Cancer DNA Methylation: Molecular Mechanisms and Clinical. Clin Cancer Res 15: 3927-3937.

5. Bird AP (1986) CpG rich islands and functions of DNA methylation. Nature 321: 209-213.

6. Adrian Bird (2002) DNA methylation patterns and epigenetic memory. Genes Dev 16: 6-21.

7. Jones PA (1999) DNA methylation paradox trends. 15: 34-37.

8. Jacqueline A, Gasche (2012) Epigenetic mechanisms in oral carcinogenesis. Future Oncol November 8(11): 1407-1425.

9. Melanie Ehrlich (2002) DNA methylation in cancer: too much, but also too little. Oncogene 21: 5400-5413.

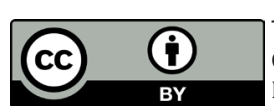

This work is licensed under Creative Commons Attribution 4.0 License DOI: $10.19080 /$ IJCSMB.2018.04.555638
10. Peter A Jones (2002) DNA methylation and cancer. Oncogene 21: 53585360.

11. Tina Branscombe Miranda, Peter A Jones (2007) DNA Methylation: The Nuts And Bolts Of Repression J Cell Physiol 213: 384-390.

12. Campo-Trapero J (2008) Update On Molecular Pathology In Oral Cancer And Precancer. Anticancer Res 28: 1197-1206.

13. Supic G, Kozomara R, Jovic N, Zeljic K, Magic Z, et al. (2011) Prognostic significance of tumor-related genes hypermethylation detected in cancer-free surgical margins of oral squamous cell carcinomas. Oral Oncol 47(8): 702-708.

14. Manel Esteller DNA methylation, Epigenetics And Metastasis.

15. Rosas SL, Koch W, Costa Carvalho MG, Wu L, Califano J, et al. (2001) Promoter hypermethylation patterns of p16, 06-methylguanine-DNAmethyltransferase, and death-associated protein kinase in tumors and saliva of head and neck cancer patients. Cancer Res 61: 939-942.

16. González-Ramírez I (2011) hMLH1 Promoter Methylation Is An Early Event In Oral Cancer. Oral Oncology 47: 22-26.

17. Zong-Chun Yi (2007) Downregulation of connexin 43 in nasopharyngeal carcinoma cells is related to promoter methylation. Oral Oncology 43: 898-904.

18. Goot Heah Khor (2013) DNA Methylation Profiling Revealed Promoter Hypermethylation-induced Silencing of p16, DDAH2 and DUSP1 in Primary Oral Squamous Cell Carcinoma. Int J Med Sci 10.

19. Shahjehan A Wajed, Laird PW, DeMeester TR (2001) DNA Methylation: An Alternative Pathway To Cancer", Ann Of Surg 234(1): 10-20.

20. Jennifer M Halbleib, James Nelson W (2006) Cadherins in development: cell adhesion, sorting, and tissue morphogenesis. Genes Dev 20: 31993214.

\section{Your next submission with Juniper Publishers} will reach you the below assets

- Quality Editorial service

- Swift Peer Review

- Reprints availability

- E-prints Service

- Manuscript Podcast for convenient understanding

- Global attainment for your research

- Manuscript accessibility in different formats ( Pdf, E-pub, Full Text, Audio)

- Unceasing customer service

Track the below URL for one-step submission https://juniperpublishers.com/online-submission.php 